\title{
Water Dynamics from THz Spectroscopy Reveal the Locus of a Liquid-Liquid Binodal Limit in Aqueous $\mathrm{CaCO}_{3}$ Solutions
}

Federico Sebastiani ${ }^{+}$, Stefan L. P. Wolf ${ }^{+}$, Benjamin Born ${ }^{+}$, Trung Quan Luong, Helmut Cölfen, Denis Gebauer,* and Martina Havenith*

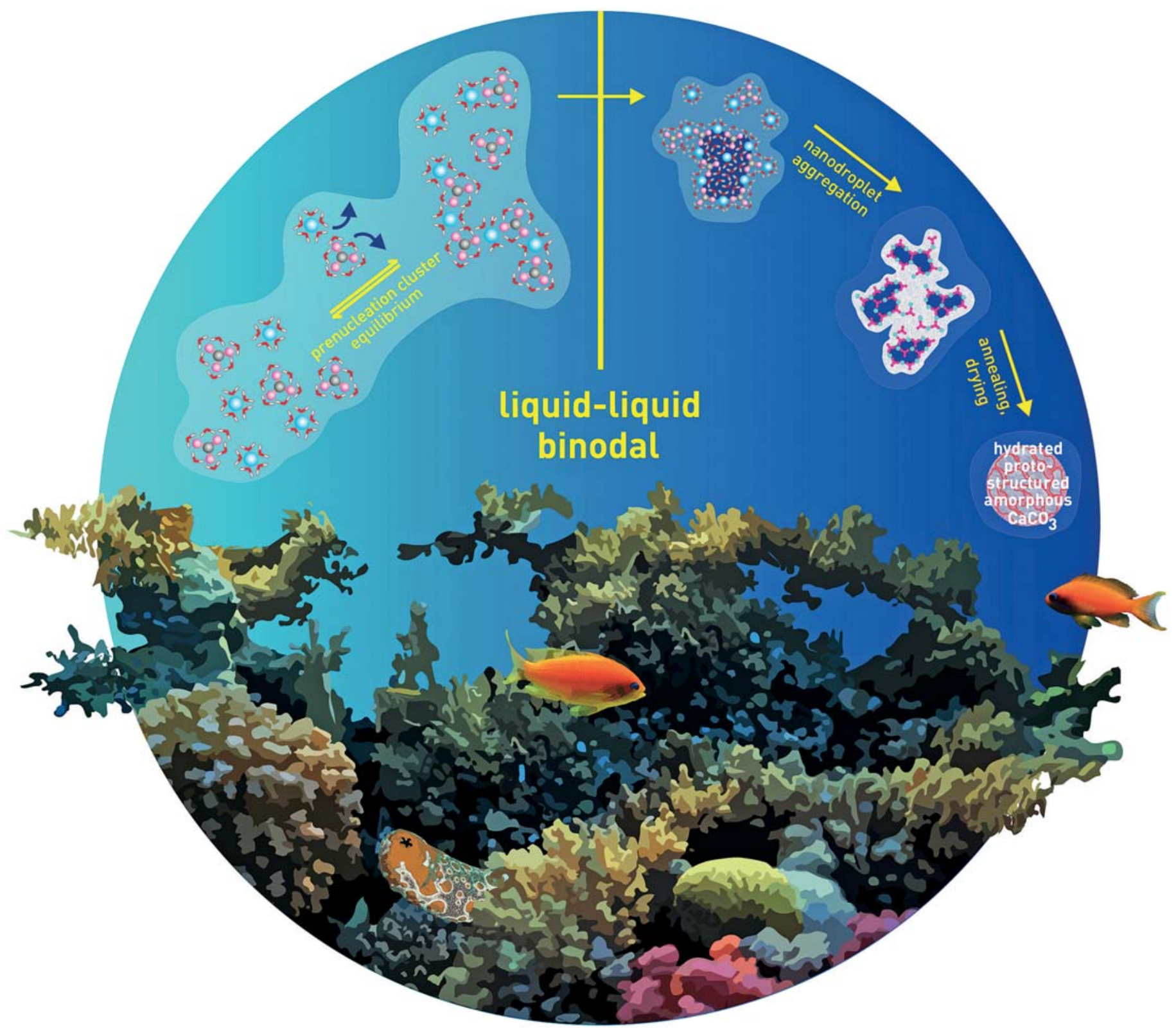




\begin{abstract}
Many phenomena depend on $\mathrm{CaCO}_{3}$ nucleation where the role of water remains enigmatic. Changes in $\mathrm{THz}$ absorption during the early stages of $\mathrm{CaCO}_{3}$ nucleation evidence altered coupled motions of hydrated calcium and carbonate ions. The direct link between these changes and the continuous development of the ion activity product reveals the locus of a liquid-liquid binodal limit. The data strongly suggest that proto-structured amorphous $\mathrm{CaCO}_{3}$ forms through solidification of initially liquid precursors. Furthermore, polycarboxylates, which stabilize liquid precursors of $\mathrm{CaCO}_{3}$, significantly enhance the kinetic stability of the metastable liquidliquid state, but they do not affect the locus of the binodal limit. The importance of water network dynamics in phase separation mechanisms can be understood based on the notions of the pre-nucleation cluster pathway, and is likely to be more general for aqueous systems.
\end{abstract}

$C_{\text {at }}$ as rock formation, ${ }^{[1]}$ biomineralization, ${ }^{[2-4]}$ or the scaling (incrustation) of pipelines. ${ }^{[5]}$ Investigations of its nucleation process and of the influence of soluble additives are relevant for the development of new materials and $\mathrm{CO}_{2}$ sequestration. ${ }^{[5-8]}$ However, the mechanism of $\mathrm{CaCO}_{3}$ nucleation is still under debate. ${ }^{[9,10]}$ It is often analyzed and interpreted from the viewpoint of classical nucleation theory $(\mathrm{CNT})^{[11,12]}$ where any role of the solvent is typically neglected. Alternatively, the so-called pre-nucleation cluster (PNC) pathway ${ }^{[9]}$ involves a stable cluster population prior to nucleation. The PNCs are regarded as solutes owing to their high dynamics and chainlike structural form called DOLLOP (dynamically ordered liquid-like oxyanion polymers $)^{[13]}$ whereas the release of water molecules from the ion hydration layers drives PNC formation. ${ }^{[14]}$ Computer simulations suggest that upon reaching a certain critical ion activity product (IAP), the PNCs can internally develop higher coordination numbers than in the initial chain-like form, slowing down the dynamics. ${ }^{[13,15]}$ This was considered as microscopic evidence for liquid-liquid demixing. ${ }^{[9,16]}$ The loci of the corresponding binodal and spinodal limits, however, beyond which phase separation can proceed with a certain barrier and proceeds spontaneously,

[*] Dr. F. Sebastiani, ${ }^{[+]}$Dr. T. Q. Luong, Prof. Dr. M. Havenith Lehrstuhl für Physikalische Chemie 2

Ruhr-Universität Bochum

Universitätsstrasse 150, 44801 Bochum (Germany)

E-mail: martina.havenith@rub.de

S. L. P. Wolf, ${ }^{[+]}$Prof. Dr. H. Cölfen, Dr. D. Gebauer

Physical Chemistry, University of Konstanz

Universitätsstrasse 10, 78457 Konstanz (Germany)

E-mail: Denis.Gebauer@uni-konstanz.de

Homepage: http://cms.uni-konstanz.de/gebauer

Dr. B. Born ${ }^{[+]}$

Department of Biological Regulation

The Weizmann Institute of Science

234 Herzl St. Rehovot, 7610001 (Israel)

$\left.{ }^{+}\right]$These authors contributed equally to this work. respectively, remain yet unknown. Upon binodal demixing, PNCs become nanodroplets that aggregate in a process driven by the decrease in the interfacial surface area. These phases can solidify or undergo a second nucleation event, yielding amorphous $\mathrm{CaCO}_{3}$ (ACC) and finally crystals. This pathway has the potential to unify various experimental observations, especially the occurrence of liquid-like intermediates stabilized by polymers (polymer-induced liquid precursors, PILPs $)^{[17]}$ that are used in bio-inspired materials chemistry. ${ }^{[6,7]}$

The interaction of ions and PNCs with water may be the key to a better understanding of the nucleation of $\mathrm{CaCO}_{3}{ }^{[18]}$ although the comprehension of the complex interplay between water and ions on the molecular scale is still a scientific challenge, despite extensive research. ${ }^{[19]} \mathrm{THz}$ spectroscopy has been shown to be a powerful tool to assess ion hydration and ion pair formation in aqueous environments. ${ }^{[20-23]}$ Aside from $\mathrm{THz}$ spectroscopic investigations of the nucleation stages of additive-free aqueous $\mathrm{CaCO}_{3}$ systems, the effect of poly(aspartic acid) (PAsp) and poly(acrylic acid) (PAA) was explored, which are known to stabilize PILPs. ${ }^{[24,6]}$ Combined with a quantitative titration assay, ${ }^{[25]}$ this approach reveals the impact of water dynamics on the early stages of $\mathrm{CaCO}_{3}$ precipitation, that is, the binodal limit according to the PNC pathway, ${ }^{[9]}$ and the influence of polycarboxylates.

The dielectric response $(\varepsilon(\omega))$ of samples prepared by subsequent mixing of $\mathrm{CaCl}_{2}, \mathrm{NaOH}$, and sodium (bi)carbonate buffer solutions was measured by $\mathrm{THz}$ time-domain spectroscopy (THz-TDS) in the frequency region of $0.2-$ $1.4 \mathrm{THz}\left(6.7-46.7 \mathrm{~cm}^{-1}\right)$ and analyzed by a double Debye relaxation model (see the Section on THz spectroscopy in the Supporting Information and Figures S1 and S2) [Eq. (1)]:[26-31]

$\varepsilon(\omega)=\varepsilon^{\prime}(\omega)-i \varepsilon^{\prime \prime}(\omega)=\varepsilon_{\infty}+\frac{\Delta \varepsilon_{1}}{1-i \omega \tau_{1}}+\frac{\Delta \varepsilon_{2}}{1-i \omega \tau_{2}}$

where $\tau_{\mathrm{i}}$ is the time constant for the $i$ th relaxation mode, $\omega$ is the frequency, $\varepsilon^{\prime}$ and $\varepsilon^{\prime \prime}$ are the real and imaginary parts of the complex dielectric constant, respectively, and $\varepsilon_{\infty}$ is the dielectric constant in the high-frequency limit. The relaxation constant $\tau_{1}$ is associated with the cooperative collective reorientation of ensembles of water molecules (ca. 8 ps for pure water). The constant for the faster relaxation, $\tau_{2}$, is usually assigned to the rotation or translation of water molecules that are not or scarcely involved in hydrogen bonds. The latter constant is in the range of $0.1-0.45$ ps for pure water, and its amplitude contributes a few percent to the overall dielectric response. ${ }^{[26-31]}$ Therefore, we restrict our discussion to $\tau_{1}$ only. At $\mathrm{pH} 9.00, \tau_{1}$ decreases significantly during the precipitation process (Figure 1), indicating a perturbation of the cooperative relaxation of the hydrogen-bond network, similar to observations made for confined water in organic solvents and micelles. ${ }^{[32,33]}$ The magnitude of this effect is in qualitative agreement with predictions from molecular dynamics (MD) simulations on another salt solution at low concentrations ${ }^{[34,35]}$ but a quantitative comparison is not possible as such effects are ion-specific. In reverse micelles, for example, the decrease in $\tau_{1}$ correlates with a decrease in the size of the micelles that is due to the 


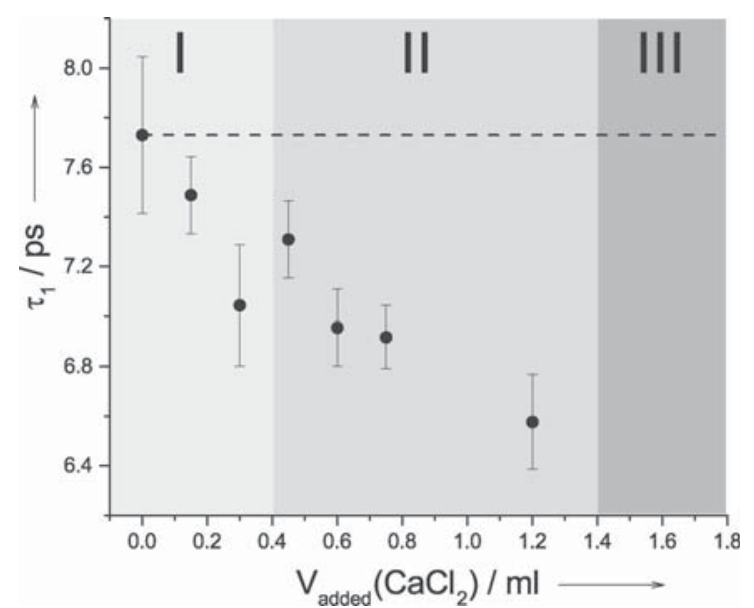

Figure 1. Relaxation constants $\left(\tau_{1}\right)$ obtained from a fit of experimental data to the double Debye model, describing the complex dielectric constant at $\mathrm{pH} 9.00$ (black circles) and of the buffer (black dashed line). The error bars represent $+/-1 \sigma$-standard deviation of $N=5$ single measurements. The $\mathrm{CaCl}_{2}$ volumes can be contrasted with the stages of precipitation in Figure $2 \mathrm{~A}$.

retardation of the water dynamics upon confinement and interfacial hydrogen bonding.

The THz-TDS data were complemented by $\mathrm{THz}$ narrowband absorption measurements (see the Section on $\mathrm{THz}$ spectroscopy experiments in the Supporting Information) in the frequency range of $2.1-2.8 \mathrm{THz}\left(\mathrm{ca} .70-95 \mathrm{~cm}^{-1}\right)$, where collective water hydrogen-bond network dynamics dominate. ${ }^{[36]}$ Distinct solution stages were prepared by continuous and slow addition of dilute $\mathrm{CaCl}_{2}$ solution into carbonate buffer at constant $\mathrm{pH}$, and identified by determining the calcium carbonate IAP with a $\mathrm{Ca}^{2+}$ ion selective electrode (under- and supersaturated pre-nucleation and early postnucleation stages, i.e., regimes I, II, and III, respectively, in Figure 2A). The corresponding developments of the difference in the absorption between the $\mathrm{CaCO}_{3}$ solutions and bulk water $(\Delta \alpha)$ at a constant $\mathrm{pH}$ value of 9.00 (Figure $2 \mathrm{~B}$ ) and pH 10.0 (Figure S3B) are similar. However, the changes in the $\mathrm{THz}$ absorption are larger at $\mathrm{pH} 9.00$ than at $\mathrm{pH} 10.0$, which is due to the larger fraction of carbonate ions in the buffer at $\mathrm{pH}$ 10.0. As much fewer calcium ions need to be added at $\mathrm{pH} 10.0$ than at $\mathrm{pH} 9.00$ to reach the same IAP, the overall concentration of ions and clusters at the distinct nucleation stages becomes significantly lower with increasing $\mathrm{pH},{ }^{[37]}$ which leads to a lower contrast.

Below the level of saturation with respect to proto-calcite $\mathrm{ACC}^{[38]}$ (region I in Figure 2B) until roughly the ACC saturation threshold, the $\mathrm{THz}$ response increases linearly, but when the system becomes supersaturated with respect to the proto-structured ACCs (regime II), ${ }^{[39]}$ it drops to a plateau at both pH 9.00 (Figure 2B) and pH 10.0 (Figure S3 B). The corresponding activity-based solubility products for protocalcite and proto-vaterite ACC of $3.1 \times 10^{-8} \mathrm{M}^{2}$ and $3.8 \times$ $10^{-8} \mathrm{M}^{2}$ at $\mathrm{pH} 9.00$ and $\mathrm{pH} 10.0$, respectively, are in accord with the literature within experimental accuracy. ${ }^{[40]}$

Regarding the increase in the absorption at $2.1-2.8 \mathrm{THz}$ in the pre-nucleation stage (undersaturated with respect to
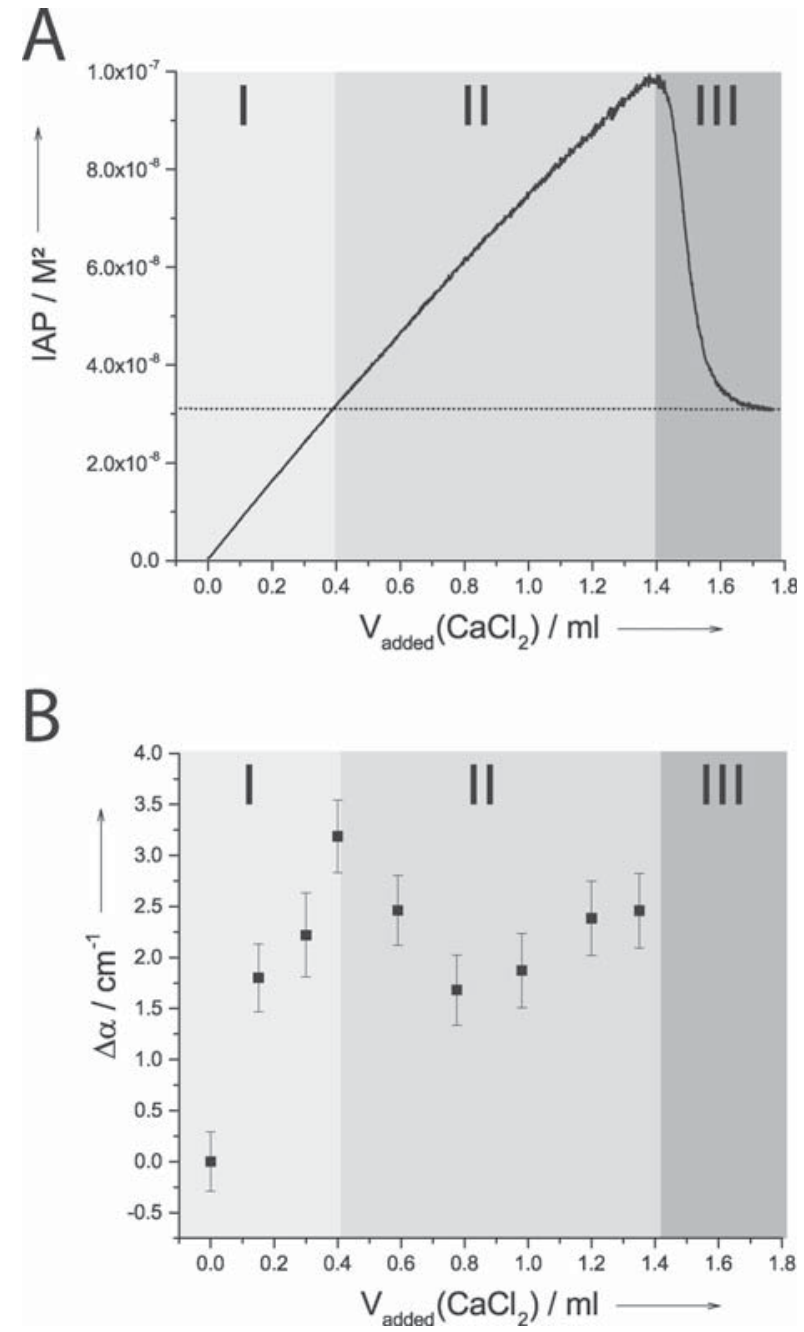

Figure 2. A) Titration data and $\mathrm{B}) \mathrm{THz}$ absorption in the frequency range of 2.1-2.8 THz without additives at $\mathrm{pH} 9.00$; the concentration of the added $\mathrm{CaCl}_{2}$ solution was $10 \mathrm{~mm}$. The horizontal dashed line $(\cdots .$.$) in (A) represents the solubility threshold of proto-calcite ACC.$ The error bars represent $+/-1 \sigma$-standard deviation of $N=4$ single measurements.

proto-calcite ACC; regime I, Figure 2), the corresponding increase in the IAP (Figure 2A) is deceptive. Upon addition of $\mathrm{CaCl}_{2}$ solution to the carbonate buffer, the total ion concentration (i.e., regimes I and II) stays virtually constant at approximately $2.0 \times 10^{-2} \mathrm{M}$ during the pre-nucleation stage, increasing by only $4.8 \times 10^{-5} \mathrm{M}$ upon entering regime II (i.e., after addition of ca. $0.4 \mathrm{~mL} \mathrm{CaCl}_{2}$ solution; Figure S4). At the same time, the free calcium concentration increases from initially zero to $7.3 \times 10^{-5} \mathrm{M}$. The corresponding increase in the $\mathrm{THz}$ absorption thus cannot be attributed to the increasing calcium ion concentration. In fact, in previous work on $\mathrm{CaCl}_{2}$ solutions, ${ }^{[20]}$ a linear $\mathrm{THz}$ absorption change on the order of $70 \mathrm{~cm}^{-1} \mathrm{M}^{-1}$ was observed and assigned to rattling modes of anions and cations within their hydration cages. Here the corresponding change would be less than $0.01 \mathrm{~cm}^{-1}$ owing to the low concentrations used. We also recorded the absorption of carbonate buffer solution and compared it to that of water $\left(\Delta \alpha=0.6 \pm 0.2 \mathrm{~cm}^{-1}\right)$. Thus the reason for the increase in $\mathrm{THz}$ 
absorption remains unclear, but we speculate that it is due to PNC formation (see below). The subsequent nonlinear response that is observed as soon as regime II is entered (Figure 2B and Figure S3 B for $\mathrm{pH} 9.00$ and $\mathrm{pH}$ 10.0, respectively) also cannot be attributed to any single-ion contributions. ${ }^{[20]}$ Both the THz-TDS and THz narrow-band absorption data sets provide strong evidence for a structural and dynamic transition in the aqueous $\mathrm{CaCO}_{3}$ system, occurring at an IAP corresponding to the solubility product of the proto-structured ACCs. This occurs long before the drop in the IAP in regime III (Figure 2A) that indicates the formation of a solid $\mathrm{CaCO}_{3}$ phase. All absorption changes are typical of the formation of neutral species rather than highly charged ionic complexes, which would give rise to more dramatic differences in the absorption, $\Delta \alpha \cdot{ }^{[20-23]}$ Importantly, the turnover in $\mathrm{THz}$ absorption is not due to the induction times associated with the formation of solid $\mathrm{CaCO}_{3}$. Experiments in which the addition of $\mathrm{CaCl}_{2}$ was stopped at defined IAPs show that the induction time after the addition of $0.8 \mathrm{~mL} \mathrm{CaCl}_{2}(10 \mathrm{~mm}$, minimum in Figure 2B) still exceeds $60 \mathrm{~min}$; however, solid $\mathrm{CaCO}_{3}$ has likely nucleated during the $\mathrm{THz}$ analysis from the sample drawn from the titration assay after addition of $1.0 \mathrm{~mL}$ $\mathrm{CaCl}_{2}$ solution (Figure S5 A).

In the presence of $5 \mu \mathrm{g} \mathrm{mL}^{-1}$ PAsp or PAA at $\mathrm{pH} 9.00$, nucleation of solid $\mathrm{CaCO}_{3}$ is strongly inhibited (Figure $3 \mathrm{~A}$ and Figure $\mathrm{S} 6 \mathrm{~A}$ ), in accordance with previous investigations. ${ }^{[41,42]}$ To ensure a similar volume dilution as in the additive-free experiments, the concentration of the $\mathrm{CaCl}_{2}$ solution was increased by a factor of ten in the presence of the polymers. The polymers do not exert a detectable effect on the $\mathrm{THz}$ absorption owing to their very low concentrations (data not shown). Again, an assessment of the induction time shows that even after the addition of $0.6 \mathrm{~mL} \mathrm{CaCl}_{2}$ solution (100 mm; Figure S5B), nucleation of solid $\mathrm{CaCO}_{3}$ does not occur immediately while the decrease in the IAP occurs very slowly, if at all. However, precipitation of solid $\mathrm{CaCO}_{3}$ likely started during the $\mathrm{THz}$ measurements after the addition of $1.0 \mathrm{~mL} 100 \mathrm{~mm} \mathrm{CaCl}$ solution in the presence of the polymers. With PAsp and PAA, the increase in $\mathrm{THz}$ absorption in regime $I$ is similar to the additive-free case, despite a larger increase in the total ion concentration. This likely arises from cooperative effects, such as the formation of neutral aggregates of ions, PNCs, and the polyanions. With $5 \mu \mathrm{mL}^{-1}$ PAsp (Figure $3 \mathrm{~A}$, see Figure S6 A for PAA), the features of the $\mathrm{THz}$ curve are somewhat more pronounced (Figure 3B) than without the polymer (Figure 2B). The turnover point of the water dynamics is not affected by the presence of either polymer (Figure S6B) although the nucleation of solid $\mathrm{CaCO}_{3}$ is strongly inhibited (transition from regime II to regime III). Starting from the addition of $1.4 \mathrm{~mL} \mathrm{CaCl}_{2}$ solution, we found a plateau in the $\mathrm{THz}$ absorption, which implies that no further changes occur with respect to the collective modes of the hydrogen-bond network.

The THz results can be understood within the notions of the so-called PNC pathway, which was proposed on the basis of extensive previous work (Figure 4) ${ }^{[9]}$ In regime I, stable PNCs (DOLLOP structural form) are in equilibrium with the free ions, and can be considered as polycondensation

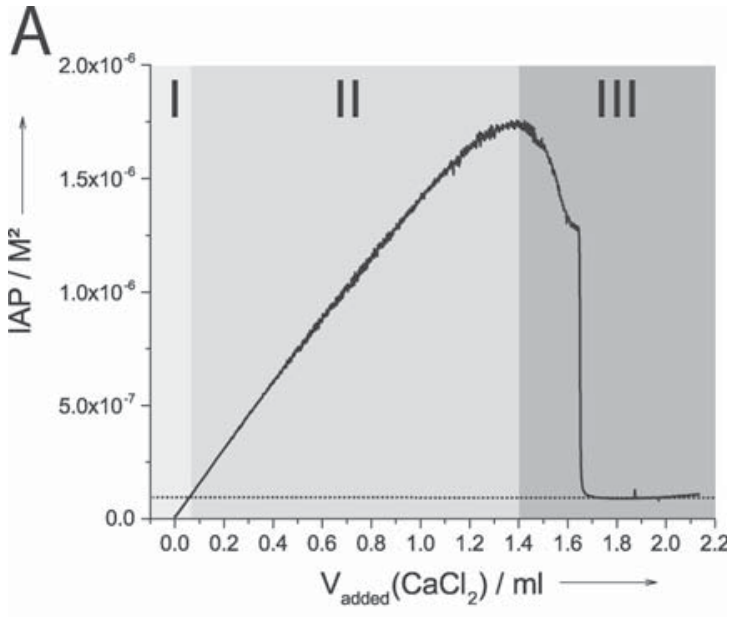

B

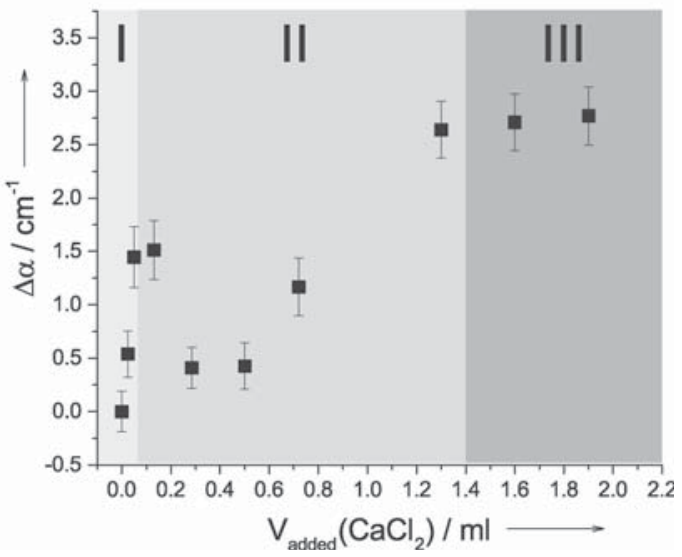

Figure 3. A) Titration curve and $\mathrm{B}$ ) $\mathrm{THz}$ absorption in the frequency range of 2.1-2.8 THz with $5 \mu \mathrm{g} \mathrm{mL}^{-1} \mathrm{PAsp}$ at $\mathrm{pH} 9.00$; the concentration of the added $\mathrm{CaCl}_{2}$ was $100 \mathrm{~mm}$. The horizontal dashed line (....•) in (A) represents the solubility threshold of the nucleated ACC phase (ca. $3.9 \times 10^{-8} \mathrm{M}^{2}$, in accord with pv-ACC within experimental accuracy). The error bars represent $+/-1 \sigma$-standard deviation of $N=4$ single measurements.

polymers of ion pairs with an average size of $1-2 \mathrm{~nm}$, as confirmed by experimental and theoretical studies. ${ }^{[9,13]}$ Recently, temperature-dependent titration experiments, in combination with isothermal titration calorimetry and MD simulations, revealed that PNC formation is driven by entropy, which can be rationalized by the release of water molecules from ionic hydration layers upon association. ${ }^{[14]}$ The fact that PNC formation is solvent-mediated and entropy-driven might explain the distinct $\mathrm{THz}$ effect observed in regime I. Owing to their high dynamics, the PNCs are regarded as solutes within a single-phase system, similar to micelles. ${ }^{[9]}$

It has been proposed on the basis of simulations that upon reaching a certain IAP, the PNC dynamics can significantly decrease, which is caused by the increase in the coordination number relative to the initial chain-like DOLLOP form and can be understood as a liquid-liquid separation event. ${ }^{[9,15]}$ This hypothesis has not yet been assessed experimentally. However, the transition from an initial increase to the non- 


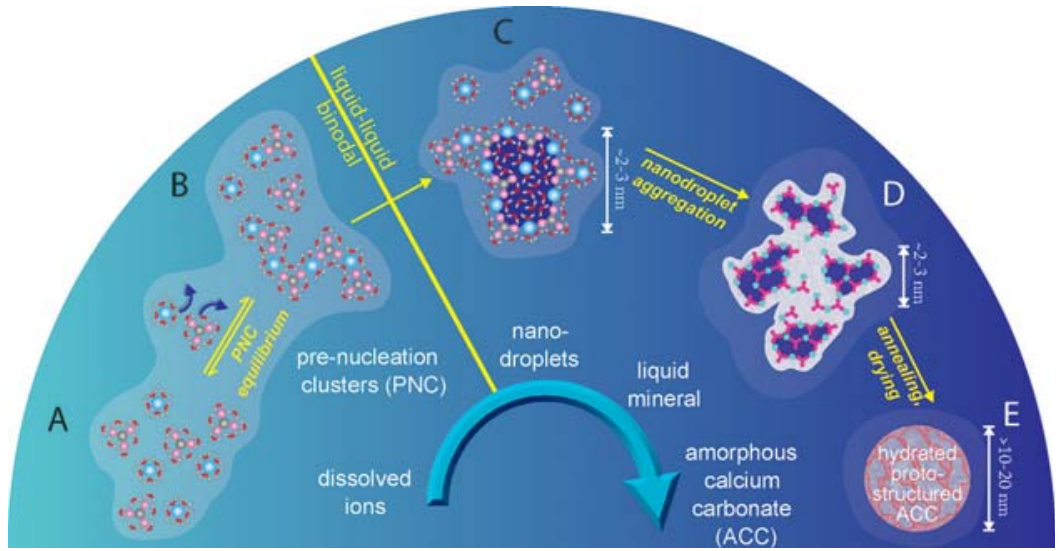

Figure 4. The role of hydration dynamics during $\mathrm{CaCO}_{3}$ nucleation. A) Hydrated calcium and carbonate ions are shown with the water molecules of their first hydration shells whereas the light blue background indicates bulk water. B) Pre-nucleation ion association is driven by a partial dehydration of the individual ions. ${ }^{[14]} \mathrm{C}$ ) Liquid-liquid separation can occur upon crossing the liquid-liquid binodal limit, and structurally and chemically corresponds to nanodroplet formation (high-density liquid) from PNCs, which are part of the homogeneous mother solution (low-density liquid). D) Aggregated nanodroplets of hydrated $\mathrm{CaCO}_{3}$ (dark blue) embedded in the network of their dynamical hydration shells (steel blue). E) Further annealing and drying results in hydrated proto-structured ACC. All sizes of the precursors and intermediates indicated in the Figure were determined elsewhere. ${ }^{[9]}$ The fact that the liquid-liquid binodal limit is governed by the stability of solid ACC strongly suggests that it is formed upon solidification of the liquid precursors as illustrated here, rather than via a second nucleation event.

linear behavior of the THz absorption (Figures 2 B and 3 B) is evidence for a change in the water dynamics. Only the coexistence of two liquid calcium carbonate phases is consistent with the continuous linear increase of the IAP at that point. ${ }^{[9]}$ Consistently, assessments of the induction times (see above) show that the observed changes in THz absorption do not correlate with the onset of precipitation of solid $\mathrm{CaCO}_{3}$. The liquid-liquid transition structurally and chemically corresponds to nanodroplet formation (high-density liquid $)^{[17]}$ from PNCs as the direct molecular precursors, which are part of the homogeneous mother solution (lowdensity liquid; Figure 4A-C; see also Figure 2A, regime II). The nanodroplets have internal pools of high-density liquid water (Figure 4C, dark blue). Subsequently, the decrease in the interfacial surface area of the droplets drives further aggregation, yielding hydrated proto-structured ACC that is approximately $10-20 \mathrm{~nm}$ in size according to previous TEM and SAXS analyses (Figure 4E; see also Figure 2, regime III). ${ }^{[39]}$ The fact that the liquid-liquid binodal limit is governed by the solubility, and thus also by the stability, of solid ACC is consistent with solidification rather than a second nucleation event that yields solids from dense liquid droplets.

In conclusion, $\mathrm{THz}$ absorption spectroscopy has been applied to observe the different nucleation stages of $\mathrm{CaCO}_{3}$, which are in accord with the notions of the PNC pathway, showing the collective dynamics of the hydrogen-bond network of the ion clusters and water molecules (see Figure 4). Common inhibitors, namely PAsp and PAA, do not modify the liquid-liquid binodal limit. Rather, organic-inorganic interactions seem to stabilize the colloidal liquid nanodrop- lets against aggregation ${ }^{[43]}$ and/or prevent their dehydration, thereby inhibiting the formation of solid $\mathrm{CaCO}_{3}$ and stabilizing the so-called PILP droplets. Our study highlights the key role of water in $\mathrm{CaCO}_{3}$ (bio)mineralization and during the nucleation process from aqueous solutions. Furthermore, we have provided fundamental information for the assessment of nucleation pathways. From the CNT point of view, the supersaturation with respect to calcite is on the order of $\ln \left(\mathrm{IAP} / K_{\text {sp }}\right) \approx 2$ (where $K_{\mathrm{sp}}$ is the calcite solubility) at the liquid-liquid binodal limit, which is in turn defined by the solubility threshold of proto-structured ACCs. The barrier for classical nucleation is tremendous under these conditions for both homogeneous and heterogeneous scenarios, ${ }^{[12]}$ rendering the occurrence of classical pathways to solid $\mathrm{CaCO}_{3}$ via critical nuclei highly improbable.

\section{Acknowledgements}

B.B. acknowledges funding from the Human Frontier Science Program (LT000336/2011). D.G. is a Research Fellow of the Zukunftskolleg of the University of Konstanz, and is supported by the Fonds der Chemischen Industrie. This work was supported by the Cluster of Excellence RESOLV (EXC 1069), which is funded by the Deutsche Forschungsgemeinschaft. We thank E. Bründermann for setting up the $\mathrm{p}$-Ge spectrometer. We are grateful to C. Hall for help with the design of the graphical material.

\section{Conflict of interest}

The authors declare no conflict of interest.

Keywords: calcium carbonate - phase diagrams .

pre-nucleation clusters $\cdot \mathrm{THz}$ spectroscopy $\cdot$ water chemistry

[1] J. Geyssant, E. Huwald, D. Strauch, Calcium Carbonate: From the Cretaceous Period into the 21st Century, Birkhäuser Verlag, Basel, Boston, 2001.

[2] J. Aizenberg, A. Tkachenko, S. Weiner, L. Addadi, G. Hendler, Nature 2001, 412, 819-822.

[3] L. Addadi, S. Raz, S. Weiner, Adv. Mater. 2003, 15, 959-970.

[4] F. C. Meldrum, H. Cölfen, Chem. Rev. 2008, 108, 4332-4432.

[5] J. Rieger, M. Kellermeier, L. Nicoleau, Angew. Chem. Int. Ed. 2014, 53, 12380-12396; Angew. Chem. 2014, 126, 12586-12603.

[6] L. B. Gower, Chem. Rev. 2008, 108, 4551-4627.

[7] J. K. Berg, T. Jordan, Y. Binder, H. G. Börner, D. Gebauer, J. Am. Chem. Soc. 2013, 135, 12512-12515. 
[8] R. E. Zeebe, J. C. Zachos, K. Caldeira, T. Tyrrell, Science 2008 $321,51-52$.

[9] D. Gebauer, M. Kellermeier, J. D. Gale, L. Bergström, H. Cölfen, Chem. Soc. Rev. 2014, 43, 2348-2371.

[10] D. Gebauer, H. Cölfen, Nano Today 2011, 6, 564-584.

[11] M. H. Nielsen, S. Aloni, J. J. De Yoreo, Science 2014, 345, 1158 1162

[12] Q. Hu et al., Faraday Discuss. 2012, 159, 509-523.

[13] R. Demichelis, P. Raiteri, J. D. Gale, D. Quigley, D. Gebauer, Nat. Commun. 2011, 2, 590.

[14] M. Kellermeier, P. Raiteri, J. Berg, A. Kempter, J. Gale, D. Gebauer, ChemPhysChem 2016, 17, 3535-3541.

[15] A. F. Wallace, L. O. Hedges, A. Fernandez-Martinez, P. Raiteri, J. D. Gale, G. A. Waychunas, S. Whitelam, J. F. Banfield, J. J. De Yoreo, Science 2013, 341, 885-889.

[16] M. Faatz, F. Gröhn, G. Wegner, Adv. Mater. 2004, 16, 996-1000.

[17] M. A. Bewernitz, D. Gebauer, J. R. Long, H. Cölfen, L. B. Gower, Faraday Discuss. 2012, 159, 291-312.

[18] P. Raiteri, J. D. Gale, J. Am. Chem. Soc. 2010, 132, 17623-17634.

[19] D. J. Tobias, J. C. Hemminger, Science 2008, 319, 1197-1198.

[20] S. Funkner, G. Niehues, D. A. Schmidt, M. Heyden, G. Schwaab, K. M. Callahan, D. J. Tobias, M. Havenith, J. Am. Chem. Soc 2012, 134, 1030-1035.

[21] V. Sharma, F. Böhm, M. Seitz, G. Schwaab, M. Havenith, Phys. Chem. Chem. Phys. 2013, 15, 8383-8391.

[22] V. Sharma, F. Böhm, G. Schwaab, M. Havenith, Phys. Chem. Chem. Phys. 2014, 16, 25101-25110.

[23] F. Böhm, V. Sharma, G. Schwaab, M. Havenith, Phys. Chem. Chem. Phys. 2015, 17, 19582-19591.

[24] L. B. Gower, D. J. Odom, J. Cryst. Growth 2000, 210, 719-734.

[25] M. Kellermeier, H. Cölfen, D. Gebauer in Methods in Enzymology, Academic Press, Burlington, 2013, pp. 45-69.

[26] C. Rønne, P.-O. Åstrand, S. R. Keiding, Phys. Rev. Lett. 1999, 82, $2888-2891$.

[27] J. T. Kindt, C. A. Schmuttenmaer, J. Phys. Chem. 1996, 100, $10373-10379$.

[28] H. Yada, M. Nagai, K. Tanaka, Chem. Phys. Lett. 2008, 464, $166-$ 170.
[29] K. J. Tielrooij, D. Paparo, L. Piatkowski, H. J. Bakker, M. Bonn, Biophys. J. 2009, 97, 2484-2492.

[30] K. J. Tielrooij, R. L. A. Timmer, H. J. Bakker, M. Bonn, Phys. Rev. Lett. 2009, 102, 198303.

[31] N. Q. Vinh, M. S. Sherwin, S. J. Allen, D. K. George, A. J. Rahmani, K. W. Plaxco, J. Chem. Phys. 2015, 142, 164502.

[32] T. Q. Luong, P. K. Verma, R. K. Mitra, M. Havenith, J. Phys. Chem. A 2011, 115, 14462-14469.

[33] A. Patra, T. Q. Luong, R. K. Mitra, M. Havenith, Phys. Chem. Chem. Phys. 2014, 16, 12875-12883.

[34] D. Laage, J. T. Hynes, Proc. Natl. Acad. Sci. USA 2007, 104, $11167-11172$.

[35] G. Stirnemann, E. Wernersson, P. Jungwirth, D. Laage, J. Am Chem. Soc. 2013, 135, 11824-11831.

[36] M. Heyden, J. Sun, S. Funkner, G. Mathias, H. Forbert, M. Havenith, D. Marx, Proc. Natl. Acad. Sci. USA 2010, 107, $12068-$ 12073.

[37] D. Gebauer, A. Völkel, H. Cölfen, Science 2008, 322, 1819-1822.

[38] D. Gebauer et al., Angew. Chem. Int. Ed. 2010, 49, 8889-8891; Angew. Chem. 2010, 122, 9073-9075.

[39] J. H. E. Cartwright, A. G. Checa, J. D. Gale, D. Gebauer, C. I. Sainz-Díaz, Angew. Chem. Int. Ed. 2012, 51, 11960-11970; Angew. Chem. 2012, 124, 12126-12137.

[40] M. Kellermeier, A. Picker, A. Kempter, H. Cölfen, D. Gebauer, Adv. Mater. 2014, 26, $752-757$.

[41] A. Verch, D. Gebauer, M. Antonietti, H. Cölfen, Phys. Chem. Chem. Phys. 2011, 13, 16811-16820.

[42] D. Gebauer, H. Cölfen, A. Verch, M. Antonietti, Adv. Mater. 2009, 21, 435-439.

[43] M. Kellermeier, D. Gebauer, E. Melero-García, M. Drechsler, Y Talmon, L. Kienle, H. Cölfen, J. M. García-Ruiz, W. Kunz, Adv. Funct. Mater. 2012, 22, $4301-4311$. 